\title{
Upper and lower limits and m-almost everywhere convergence of intuitionistic fuzzy observables
}

\author{
Katarína Čunderlíková \\ Mathematical Institute, Slovak Academy of Sciences \\ Štefánikova 49, 81473 Bratislava, Slovakia \\ e-mail: cunderlikova.lendelova@gmail.com
}

This paper is dedicated to the memory of Prof. RNDr. Beloslav Riečan, DrSc., Dr.h.c.

Received: 28 March 2018

Accepted: 7 September 2018

\begin{abstract}
In paper [4] we defined the upper and the lower limits for sequence of intuitionistic fuzzy observables with the help of intuitionistic fuzzy probability $\mathcal{P}$ and we compared two concepts of $\mathcal{P}$-almost everywhere convergence. The aim of this paper is to define the lower and upper limits using the intuitionistic fuzzy state $\mathbf{m}$. We study two concepts of $\mathbf{m}$-almost everywhere convergence and we show that they are equivalent, too.
\end{abstract}

Keywords: IF-observable, m-almost everywhere convergence, Upper limit, Lower limit, IF-sets, IF-state, IF-probability, Zero IF-observable.

2010 Mathematics Subject Classification: 03B52, 60A86, 60B10.

\section{Introduction}

In $[1,2]$ K. T. Atanassov introduced the notion of intuitionistic fuzzy sets. Then in [3] Grzegorzewski and Mrówka defined the probability on the family of intuitionistic fuzzy events

$$
\mathcal{N}=\left\{\left(\mu_{A}, \nu_{A}\right) ; \mu_{A}+\nu_{A} \leq 1\right\},
$$

where $\mu_{A}, \nu_{A}$ are $\mathcal{S}$-measurable, as a mapping $\mathcal{P}$ from the family $\mathcal{N}$ to the set of all compact intervals in $\mathbf{R}$ by the formula

$$
\mathcal{P}\left(\left(\mu_{A}, \nu_{A}\right)\right)=\left[\int_{\Omega} \mu_{A} d P, 1-\int_{\Omega} \nu_{A} d P\right],
$$


where $(\Omega, \mathcal{S}, P)$ is probability space. This IF-probability was axiomatically characterized by B. Riečan (see[5]).

In paper [4] we defined the upper and the lower limits for sequence of intuitionistic fuzzy observables with the help of intuitionistic fuzzy probability $\mathcal{P}$ and we compared two concepts of $\mathcal{P}$-almost everywhere convergence, where $\mathbf{m}$ is an intuitionistic fuzzy state. Since the intuitionistic fuzzy probability $\mathcal{P}$ can be decomposed to two intuitionistic fuzzy states, it is usefull to study $\mathbf{m}$-almost everywhere convergence. In this paper we define the lower and upper limits for a sequence of intuitionistic fuzzy observables and we study two concepts of $\mathbf{m}$-almost everywhere convergence, too.

Remark that in a whole text we use a notation "IF" for short a phrase "intuitionistic fuzzy".

\section{IF-events, IF-states and IF-observables}

Definition 2.1. Let $\Omega$ be a nonempty set. An IF-set $\mathbf{A}$ on $\Omega$ is a pair $\left(\mu_{A}, \nu_{A}\right)$ of mappings $\mu_{A}, \nu_{A}: \Omega \rightarrow[0,1]$ such that $\mu_{A}+\nu_{A} \leq 1_{\Omega}$.

Definition 2.2. Start with a measurable space $(\Omega, \mathcal{S})$. Hence $\mathcal{S}$ is a $\sigma$-algebra of subsets of $\Omega$. An IF-event is called an IF-set $\mathbf{A}=\left(\mu_{A}, \nu_{A}\right)$ such that $\mu_{A}, \nu_{A}: \Omega \rightarrow[0,1]$ are $\mathcal{S}$-measurable.

The family of all IF-events on $(\Omega, \mathcal{S})$ will be denoted by $\mathcal{F}, \mu_{A}: \Omega \longrightarrow[0,1]$ will be called the membership function, $\nu_{A}: \Omega \longrightarrow[0,1]$ be called the non-membership function.

If $\mathbf{A}=\left(\mu_{A}, \nu_{A}\right) \in \mathcal{F}, \mathbf{B}=\left(\mu_{B}, \nu_{B}\right) \in \mathcal{F}$, then we define the Łukasiewicz binary operations $\oplus, \odot$ on $\mathcal{F}$ by

$$
\begin{aligned}
& \left.\mathbf{A} \oplus \mathbf{B}=\left(\left(\mu_{A}+\mu_{B}\right) \wedge 1,\left(\nu_{A}+\nu_{B}-1\right) \vee 0\right)\right) \\
& \left.\mathbf{A} \odot \mathbf{B}=\left(\left(\mu_{A}+\mu_{B}-1\right) \vee 0,\left(\nu_{A}+\nu_{B}\right) \wedge 1\right)\right)
\end{aligned}
$$

and the partial ordering is given by

$$
\mathbf{A} \leq \mathbf{B} \Longleftrightarrow \mu_{A} \leq \mu_{B}, \nu_{A} \geq \nu_{B}
$$

In the paper we use the max-min connectives defined by

$\mathbf{A} \vee \mathbf{B}=\left(\mu_{A} \vee \mu_{B}, \nu_{A} \wedge \nu_{B}\right)$,

$\mathbf{A} \wedge \mathbf{B}=\left(\mu_{A} \wedge \mu_{B}, \nu_{A} \vee \nu_{B}\right)$

and the De Morgan rules

$$
\begin{aligned}
& (a \vee b)^{*}=a^{*} \wedge b^{*}, \\
& (a \wedge b)^{*}=a^{*} \vee b^{*}
\end{aligned}
$$

where $a^{*}=1-a$. 
Example 2.3. The fuzzy set $f: \Omega \longrightarrow[0,1]$ can be regarded as an $I F$-set, if we put

$$
\mathbf{A}=(f, 1-f) .
$$

If $f=\chi_{A}$, then the corresponding IF-set has the form

$$
\mathbf{A}=\left(\chi_{A}, 1-\chi_{A}\right)=\left(\chi_{A}, \chi_{A^{\prime}}\right)
$$

In this case $\mathbf{A} \oplus \mathbf{B}$ corresponds to the union of sets, $\mathbf{A} \odot \mathbf{B}$ to the product of sets, and $\leq$ to the set inclusion.

In the $I F$-probability theory ([7], [8]) instead of the notion of probability we use the notion of state.

Definition 2.4. Let $\mathcal{F}$ be the family of all IF-events in $\Omega$. A mapping $\mathbf{m}: \mathcal{F} \rightarrow[0,1]$ is called an IF-state, if the following conditions are satisfied:

(i) $\mathbf{m}\left(\left(1_{\Omega}, 0_{\Omega}\right)\right)=1, \mathbf{m}\left(\left(0_{\Omega}, 1_{\Omega}\right)\right)=0$;

(ii) if $\mathbf{A} \odot \mathbf{B}=\left(0_{\Omega}, 1_{\Omega}\right)$ and $\mathbf{A}, \mathbf{B} \in \mathcal{F}$, then $\mathbf{m}(\mathbf{A} \oplus \mathbf{B})=\mathbf{m}(\mathbf{A})+\mathbf{m}(\mathbf{B})$;

(iii) if $\mathbf{A}_{n} \nearrow \mathbf{A}\left(\right.$ i.e. $\left.\mu_{A_{n}} \nearrow \mu_{A}, \nu_{A_{n}} \searrow \nu_{A}\right)$, then $\mathbf{m}\left(\mathbf{A}_{n}\right) \nearrow \mathbf{m}(\mathbf{A})$.

Probably the most useful result in the $I F$-state theory is the following representation theorem ([6]):

Theorem 2.5. To each IF-state $\mathbf{m}: \mathcal{F} \rightarrow[0,1]$ there exists exactly one probability measure $P: \mathcal{S} \rightarrow[0,1]$ and exactly one $\alpha \in[0,1]$ such that

$$
\mathbf{m}(\mathbf{A})=(1-\alpha) \int_{\Omega} \mu_{A} d P+\alpha\left(1-\int_{\Omega} \nu_{A} d P\right)
$$

for each $\mathbf{A}=\left(\mu_{A}, \nu_{A}\right) \in \mathcal{F}$.

The third basic notion in the probability theory is the notion of an observable. Let $\mathcal{J}$ be the family of all intervals in $R$ of the form

$$
[a, b)=\{x \in R \mid a \leq x<b\} .
$$

Then the $\sigma$-algebra $\sigma(\mathcal{J})$ is denoted $\mathcal{B}(R)$ and it is called the $\sigma$-algebra of Borel sets, its elements are called Borel sets.

Definition 2.6. By an IF-observable on $\mathcal{F}$ we understand each mapping $x: \mathcal{B}(R) \rightarrow \mathcal{F}$ satisfying the following conditions:

(i) $x(R)=\left(1_{\Omega}, 0_{\Omega}\right), x(\emptyset)=\left(0_{\Omega}, 1_{\Omega}\right) ;$

(ii) if $A \cap B=\emptyset$, then $x(A) \odot x(B)=\left(0_{\Omega}, 1_{\Omega}\right)$ and $x(A \cup B)=x(A) \oplus x(B)$;

(iii) if $A_{n} \nearrow A$, then $x\left(A_{n}\right) \nearrow x(A)$. 
If we denote $x(A)=\left(x^{b}(A), 1-x^{\sharp}(A)\right)$ for each $A \in \mathcal{B}(R)$, then $x^{b}, x^{\sharp}: \mathcal{B}(R) \rightarrow \mathcal{T}$ are observables, where $\mathcal{T}=\{f: \Omega \rightarrow[0,1] ; f$ is $\mathcal{S}$-measurable $\}$.

Similarly as in the classical case the following theorem can be proved ([8]).

Theorem 2.7. Let $x: \mathcal{B}(R) \longrightarrow \mathcal{F}$ be an IF-observable, $\mathbf{m}: \mathcal{F} \longrightarrow[0,1]$ be an IF-state. Define the mapping $\mathbf{m}_{x}: \mathcal{B}(R) \longrightarrow[0,1]$ by the formula

$$
\mathbf{m}_{x}(C)=\mathbf{m}(x(C))
$$

Then $\mathbf{m}_{x}: \mathcal{B}(R) \longrightarrow[0,1]$ is a probability measure.

\section{Lower and upper limits}

Let $\left(\xi_{n}\right)_{n}$ be a sequence of random variables in a probability space $(\Omega, \mathcal{S}, P)$. It is easy to see that

$$
\limsup _{n \rightarrow \infty} \xi_{n}(\omega)<t \Longleftrightarrow \omega \in \bigcup_{p=1}^{\infty} \bigcup_{k=1}^{\infty} \bigcap_{n=k}^{\infty} \xi^{-1}\left(\left(-\infty, t-\frac{1}{p}\right)\right),
$$

for every $\omega \in \Omega$. This relation justifies the following definition.

Definition 3.1. We shall say that a sequence $\left(x_{n}\right)_{n}$ of IF-observables has lim sup, if there exists an IF-observable $\bar{x}: \mathcal{B}(R) \rightarrow \mathcal{F}$ such that

$$
\bar{x}((-\infty, t))=\bigvee_{p=1}^{\infty} \bigvee_{k=1}^{\infty} \bigwedge_{n=k}^{\infty} x_{n}\left(\left(-\infty, t-\frac{1}{p}\right)\right)
$$

for every $t \in R$. We write $\bar{x}=\limsup _{n \rightarrow \infty} x_{n}$.

Note that if another IF-observable y satisfies the above condition, then $\mathbf{m} \circ y=\mathbf{m} \circ \bar{x}$.

Definition 3.2. A sequence $\left(x_{n}\right)_{n}$ of IF-observables has $\liminf _{n \rightarrow \infty}$ if there exists an IF-observable x such that

$$
\underline{x}((-\infty, t))=\bigvee_{p=1}^{\infty} \bigwedge_{k=1}^{\infty} \bigvee_{n=k}^{\infty} x_{n}\left(\left(-\infty, t-\frac{1}{p}\right)\right)
$$

for all $t \in R$. Notation: $\underline{x}=\liminf _{n \rightarrow \infty} x_{n}$.

Theorem 3.3. The IF-observables $\bar{x}, \underline{x}$ from Definition 3.1 and Definition 3.2 can be expressed in the following form

$$
\begin{aligned}
& \bar{x}(A)=\left(\overline{x^{b}}(A), 1-\overline{x^{\sharp}}(A)\right), \\
& \underline{x}(A)=\left(\underline{x^{b}}(A), 1-\underline{x^{\sharp}}(A)\right),
\end{aligned}
$$

for each $A \in \mathcal{B}(R)$. Here $\overline{x^{b}}, \underline{x^{b}}$ are upper and lower limits of sequence $\left(x_{n}^{b}\right)_{1}^{\infty}$ of observables in tribe $\mathcal{T}$ and $\overline{x^{\sharp}}, \underline{x^{\sharp}}$ are upper and lower limits of sequence $\left(x_{n}^{\sharp}\right)_{1}^{\infty}$ of observables in tribe $\mathcal{T}$ (see [9]). 
Proof. Let $\left(x_{n}\right)_{1}^{\infty}$ be a sequence of IF-observables. Denote

$$
x_{n}(A)=\left(x_{n}^{b}(A), 1-x_{n}^{\sharp}(A)\right)
$$

for each $A \in \mathcal{B}(R)$, then $x_{n}^{b}, x_{n}^{\sharp}: \mathcal{B}(R) \rightarrow \mathcal{T}$ are observables for every $n \in N$.

Therefore using definition of max-min connectives $\vee, \wedge$ and the De Morgan rules, we obtain

$$
\begin{aligned}
\bar{x}(-\infty, t) & =\bigvee_{p=1}^{\infty} \bigvee_{k=1}^{\infty} \bigwedge_{n=k}^{\infty} x_{n}\left(-\infty, t-\frac{1}{p}\right)= \\
& =\left(\bigvee_{p=1}^{\infty} \bigvee_{k=1}^{\infty} \bigwedge_{n=k}^{\infty} x_{n}^{b}\left(-\infty, t-\frac{1}{p}\right), 1-\bigvee_{p=1}^{\infty} \bigvee_{k=1}^{\infty} \bigwedge_{n=k}^{\infty} x_{n}^{\sharp}\left(-\infty, t-\frac{1}{p}\right)\right)= \\
& =\left(\overline{x^{b}}(-\infty, t), 1-\overline{x^{\sharp}}(-\infty, t)\right)
\end{aligned}
$$

and

$$
\begin{aligned}
\underline{x}(-\infty, t) & =\bigvee_{p=1}^{\infty} \bigwedge_{k=1}^{\infty} \bigvee_{n=k}^{\infty} x_{n}\left(-\infty, t-\frac{1}{p}\right)= \\
& =\left(\bigvee_{p=1}^{\infty} \bigwedge_{k=1}^{\infty} \bigvee_{n=k}^{\infty} x_{n}^{b}\left(-\infty, t-\frac{1}{p}\right), 1-\bigvee_{p=1}^{\infty} \bigwedge_{k=1}^{\infty} \bigvee_{n=k}^{\infty} x_{n}^{\sharp}\left(-\infty, t-\frac{1}{p}\right)\right)= \\
& =\left(\underline{x^{b}}(-\infty, t), 1-\underline{x^{\sharp}}(-\infty, t)\right)
\end{aligned}
$$

for every $t \in R$.

Proposition 3.1. Let $\left(x_{n}\right)_{n}$ be a bounded sequence of IF-observables, i.e., there exist IF-observables $y, z: \mathcal{B}(R) \rightarrow \mathcal{F}$ such that

$$
y((-\infty, t)) \leq x_{n}((-\infty, t)) \leq z((-\infty, t))
$$

for every $t \in R$ and every $n \in N$. Then there exists $\limsup _{n \rightarrow \infty} x_{n}$.

Proof. Since $y, x_{n}, z$ are IF-observables, then we can write them in the following form

$$
\begin{aligned}
y((-\infty, t)) & =\left(y^{b}((-\infty, t)), 1-y^{\sharp}((-\infty, t))\right), \\
x_{n}((-\infty, t)) & =\left(x_{n}^{b}((-\infty, t)), 1-x_{n}^{\sharp}((-\infty, t))\right), \\
z((-\infty, t)) & =\left(z^{b}((-\infty, t)), 1-z^{\sharp}((-\infty, t))\right),
\end{aligned}
$$

where $y^{b}, y^{\sharp}, x_{n}^{b}, x_{n}^{\sharp}, z^{b}, z^{\sharp}: \mathcal{B}(R) \rightarrow \mathcal{T}$ are observables.

Such

$$
y((-\infty, t)) \leq x_{n}((-\infty, t)) \leq z((-\infty, t))
$$

then

$\left(y^{b}((-\infty, t)), 1-y^{\sharp}((-\infty, t))\right) \leq\left(x_{n}^{b}((-\infty, t)), 1-x_{n}^{\sharp}((-\infty, t))\right) \leq\left(z^{b}((-\infty, t)), 1-z^{\sharp}((-\infty, t))\right)$.

Hence

$$
y^{b}((-\infty, t)) \leq x_{n}^{b}((-\infty, t)) \leq z^{b}((-\infty, t)),
$$




$$
\begin{gathered}
1-y^{\sharp}((-\infty, t)) \geq 1-x_{n}^{\sharp}((-\infty, t)) \geq 1-z^{\sharp}((-\infty, t)), \\
y^{\sharp}((-\infty, t)) \leq x_{n}^{\sharp}((-\infty, t)) \leq z^{\sharp}((-\infty, t))
\end{gathered}
$$

for every $t \in R$ and every $n \in N$.

Using Proposition 8.6.2 from [9] then there exist upper limits $\overline{x^{b}}, \overline{x^{\sharp}}: \mathcal{B}(R) \rightarrow \mathcal{T}$. Finally, from Theorem 3.3 we obtain that there exists $\bar{x}=\limsup x_{n}$ given by

$$
\bar{x}((-\infty, t))=\left(\overline{x^{b}}((-\infty, t)), 1-\overline{x^{\sharp}}((-\infty, t))\right) .
$$

Proposition 3.2. If a sequence of IF-observables $\left(x_{n}\right)_{n}$ has $\bar{x}=\limsup _{n \rightarrow \infty} x_{n}$ and $\underline{x}=\liminf _{n \rightarrow \infty} x_{n}$, then

$$
\bar{x}((-\infty, t)) \leq \underline{x}((-\infty, t)),
$$

for every $t \in R$

Proof. Let a sequence of IF-observables $\left(x_{n}\right)_{n}$ has $\bar{x}, \underline{x}$. Then by Theorem 3.3 there exist observables $\overline{x^{b}}, \underline{x^{b}}, \overline{x^{\sharp}}, \underline{x^{\sharp}}$ in tribe $\mathcal{T}$, such that

$$
\begin{aligned}
& \bar{x}((-\infty, t))=\left(\overline{x^{b}}((-\infty, t)), 1-\overline{x^{\sharp}}((-\infty, t))\right), \\
& \underline{x}((-\infty, t))=\left(\underline{x^{b}}((-\infty, t)), 1-\underline{x^{\sharp}}((-\infty, t))\right) .
\end{aligned}
$$

Hence by Proposition 8.6.4 in [9] we have the following inequalities for every $t \in R$

$$
\begin{aligned}
& \overline{x^{b}}((-\infty, t)) \leq \underline{x^{b}}((-\infty, t)), \\
& \overline{x^{\sharp}}((-\infty, t)) \leq \underline{x^{\sharp}}((-\infty, t)),
\end{aligned}
$$

then we obtain

$$
1-\overline{x^{\sharp}}((-\infty, t)) \geq 1-\underline{x^{\sharp}}((-\infty, t))
$$

by simple modifications.

Therefore, by (1), (2) and Theorem 3.3 we have

$$
\bar{x}((-\infty, t)) \leq \underline{x}((-\infty, t)) .
$$

\section{Almost everywhere convergence}

Recall that a sequence $\left(\xi_{n}\right)_{n}$ of random variables converges almost everywhere to 0 , if

$$
P\left(\bigcap_{p=1}^{\infty} \bigcup_{k=1}^{\infty} \bigcap_{n=k}^{\infty} \xi_{n}^{-1}\left(\left(-\frac{1}{p}, \frac{1}{p}\right)\right)\right)=\lim _{n \rightarrow \infty} P\left(\bigcap_{n=k}^{k+i} \xi_{n}^{-1}\left(\left(-\frac{1}{p}, \frac{1}{p}\right)\right)\right)=1 .
$$

Similarly we can work with a sequence of $I F$-observables on $(\mathcal{F}, \mathbf{m})$.

Definition 4.1. Let $\left(x_{n}\right)_{n}$ be a sequence of $I F$-observables on an $I F$-space $(\mathcal{F}, \mathbf{m})$. We say that $\left(x_{n}\right)_{n}$ converges $\mathbf{m}$-almost everywhere to 0 , if

$$
\mathbf{m}\left(\bigwedge_{p=1}^{\infty} \bigvee_{k=1}^{\infty} \bigwedge_{n=k}^{\infty} x_{n}\left(\left(-\frac{1}{p}, \frac{1}{p}\right)\right)\right)=\lim _{n \rightarrow \infty} \mathbf{m}\left(\bigwedge_{n=k}^{k+i} x_{n}\left(\left(-\frac{1}{p}, \frac{1}{p}\right)\right)\right)=1
$$


Remark 4.2. The defining formula is equivalent to the following equality

$$
\mathbf{m}\left(\bigvee_{p=1}^{\infty} \bigwedge_{k=1}^{\infty} \bigvee_{n=k}^{\infty} x_{n}\left(R \backslash\left(-\frac{1}{p}, \frac{1}{p}\right)\right)\right)=0
$$

Definition 4.3. By the zero IF-observable $0_{\mathcal{F}}$ we shall denote the IF-observable defined by the following formula

$$
0_{\mathcal{F}}((-\infty, t))= \begin{cases}(1,0), & \text { if } t>0 \\ (0,1), & \text { if } t \leq 0\end{cases}
$$

for each $t \in R$.

Remark 4.4. The zero IF-observable $0_{\mathcal{F}}$ can be rewritten in the following form

$$
0_{\mathcal{F}}(A)= \begin{cases}(1,0), & \text { if } 0 \in A \\ (0,1), & \text { if } 0 \notin A\end{cases}
$$

for each $A=(-\infty, t)$ and $t \in R$.

Proposition 4.1. A sequence $\left(x_{n}\right)_{n}$ of IF-observables converges $\mathbf{m}$-almost everywhere to 0 if and only if

$$
\mathbf{m}\left(\bigvee_{p=1}^{\infty} \bigvee_{k=1}^{\infty} \bigwedge_{n=k}^{\infty} x_{n}\left(\left(-\infty, t-\frac{1}{p}\right)\right)\right)=\mathbf{m}\left(\bigvee_{p=1}^{\infty} \bigwedge_{k=1}^{\infty} \bigvee_{n=k}^{\infty} x_{n}\left(\left(-\infty, t-\frac{1}{p}\right)\right)\right)=\mathbf{m}\left(0_{\mathcal{F}}((-\infty, t))\right)
$$

for every $t \in R$.

Proof. " $\Rightarrow$ " Let a sequence $\left(x_{n}\right)_{n}$ of an $I F$-observables converges $\mathbf{m}$-almost everywhere to 0 . Then from Definition 4.1 we have

$$
\mathbf{m}\left(\bigwedge_{p=1}^{\infty} \bigvee_{k=1}^{\infty} \bigwedge_{n=k}^{\infty} x_{n}\left(\left(-\frac{1}{p}, \frac{1}{p}\right)\right)\right)=\lim _{n \rightarrow \infty} \mathbf{m}\left(\bigwedge_{n=k}^{k+i} x_{n}\left(\left(-\frac{1}{p}, \frac{1}{p}\right)\right)\right)=1
$$

i.e.,

$$
\mathbf{m}\left(\bigvee_{p=1}^{\infty} \bigwedge_{k=1}^{\infty} \bigvee_{n=k}^{\infty} x_{n}\left(R \backslash\left(-\frac{1}{p}, \frac{1}{p}\right)\right)\right)=0
$$

Since $R \backslash\left(-\frac{1}{p}, \frac{1}{p}\right)=\left(-\infty,-\frac{1}{p}\right] \cup\left[\frac{1}{p}, \infty\right)$, then

$$
x_{n}\left(R \backslash\left(-\frac{1}{p}, \frac{1}{p}\right)\right)=x_{n}\left(\left(-\infty,-\frac{1}{p}\right]\right) \oplus x_{n}\left(\left[\frac{1}{p}, \infty\right)\right) .
$$

For $t \leq 0$ is $\left(-\infty, t-\frac{1}{p}\right) \subset\left(-\infty,-\frac{1}{p}\right) \subset R \backslash\left(-\frac{1}{p}, \frac{1}{p}\right)$, hence

$$
x_{n}\left(\left(-\infty, t-\frac{1}{p}\right)\right) \leq x_{n}\left(\left(-\infty,-\frac{1}{p}\right)\right) \leq x_{n}\left(R \backslash\left(-\frac{1}{p}, \frac{1}{p}\right)\right)
$$

and therefore

$$
\mathbf{m}\left(\bigvee_{n=k}^{k+i} x_{n}\left(\left(-\infty, t-\frac{1}{p}\right)\right)\right) \leq \mathbf{m}\left(\bigvee_{n=k}^{k+i} x_{n}\left(R \backslash\left(-\frac{1}{p}, \frac{1}{p}\right)\right)\right) .
$$


Finally from Proposition 3.2, (4) and (5) we obtain

$$
\begin{aligned}
0 \leq \mathbf{m}\left(\bigvee_{p=1}^{\infty} \bigvee_{k=1}^{\infty} \bigwedge_{n=k}^{\infty} x_{n}\left(\left(-\infty, t-\frac{1}{p}\right)\right)\right) & \leq \mathbf{m}\left(\bigvee_{p=1}^{\infty} \bigwedge_{k=1}^{\infty} \bigvee_{n=k}^{\infty} x_{n}\left(\left(-\infty, t-\frac{1}{p}\right)\right)\right) \leq \\
& \leq \mathbf{m}\left(\bigvee_{p=1}^{\infty} \bigwedge_{k=1}^{\infty} \bigvee_{n=k}^{\infty} x_{n}\left(R \backslash\left(-\frac{1}{p}, \frac{1}{p}\right)\right)\right)=0 .
\end{aligned}
$$

But $\mathbf{m}\left(0_{\mathcal{F}}((-\infty, t))\right)=0$ for $t \leq 0$, hence

$$
\mathbf{m}\left(\bigvee_{p=1}^{\infty} \bigvee_{k=1}^{\infty} \bigwedge_{n=k}^{\infty} x_{n}\left(\left(-\infty, t-\frac{1}{p}\right)\right)\right)=\mathbf{m}\left(\bigvee_{p=1}^{\infty} \bigwedge_{k=1}^{\infty} \bigvee_{n=k}^{\infty} x_{n}\left(\left(-\infty, t-\frac{1}{p}\right)\right)\right)=\mathbf{m}\left(0_{\mathcal{F}}((-\infty, t))\right)
$$

On the other hand, if $t>0$, then there exists $p$ such that $\left(-\frac{1}{p}, \frac{1}{p}\right) \subset\left(-\infty, t-\frac{1}{p}\right)$. Therefore

$$
x_{n}\left(\left(-\frac{1}{p}, \frac{1}{p}\right)\right) \leq x_{n}\left(\left(-\infty, t-\frac{1}{p}\right)\right)
$$

and hence

$$
\mathbf{m}\left(\bigwedge_{n=k}^{k+i} x_{n}\left(\left(-\frac{1}{p}, \frac{1}{p}\right)\right)\right) \leq \mathbf{m}\left(\bigwedge_{n=k}^{k+i} x_{n}\left(\left(-\infty, t-\frac{1}{p}\right)\right)\right)
$$

Finally from Proposition 3.2, (3) and (6) we obtain

$$
\begin{aligned}
1=\mathbf{m}\left(\bigwedge_{p=1}^{\infty} \bigvee_{k=1}^{\infty} \bigwedge_{n=k}^{\infty} x_{n}\left(\left(-\frac{1}{p}, \frac{1}{p}\right)\right)\right) & \leq \mathbf{m}\left(\bigvee_{p=1}^{\infty} \bigvee_{k=1}^{\infty} \bigwedge_{n=k}^{\infty} x_{n}\left(\left(-\infty, t-\frac{1}{p}\right)\right)\right) \leq \\
& \leq \mathbf{m}\left(\bigvee_{p=1}^{\infty} \bigwedge_{k=1}^{\infty} \bigvee_{n=k}^{\infty} x_{n}\left(\left(-\infty, t-\frac{1}{p}\right)\right)\right) \leq 1
\end{aligned}
$$

But $\mathbf{m}\left(0_{\mathcal{F}}((-\infty, t))\right)=1$ for $t>0$, hence

$$
\mathbf{m}\left(\bigvee_{p=1}^{\infty} \bigvee_{k=1}^{\infty} \bigwedge_{n=k}^{\infty} x_{n}\left(\left(-\infty, t-\frac{1}{p}\right)\right)\right)=\mathbf{m}\left(\bigvee_{p=1}^{\infty} \bigwedge_{k=1}^{\infty} \bigvee_{n=k}^{\infty} x_{n}\left(\left(-\infty, t-\frac{1}{p}\right)\right)\right)=\mathbf{m}\left(0_{\mathcal{F}}((-\infty, t))\right)
$$

“Е” Let $\mathbf{m}\left(\bigvee_{p=1}^{\infty} \bigvee_{k=1}^{\infty} \bigwedge_{n=k}^{\infty} x_{n}\left(\left(-\infty, t-\frac{1}{p}\right)\right)\right)=\mathbf{m}\left(\bigvee_{p=1}^{\infty} \bigwedge_{k=1}^{\infty} \bigvee_{n=k}^{\infty} x_{n}\left(\left(-\infty, t-\frac{1}{p}\right)\right)\right)=$ $\mathbf{m}\left(0_{\mathcal{F}}((-\infty, t))\right)$ for every $t \in R$. We have to prove that a sequence $\left(x_{n}\right)_{n}$ of an $I F$-observables converges $\mathbf{m}$-almost everywhere to 0 , i.e.

$$
\mathbf{m}\left(\bigwedge_{p=1}^{\infty} \bigvee_{k=1}^{\infty} \bigwedge_{n=k}^{\infty} x_{n}\left(\left(-\frac{1}{p}, \frac{1}{p}\right)\right)\right)=1
$$

what is equivalent with

$$
\mathbf{m}\left(\bigvee_{p=1}^{\infty} \bigwedge_{k=1}^{\infty} \bigvee_{n=k}^{\infty} x_{n}\left(R \backslash\left(-\frac{1}{p}, \frac{1}{p}\right)\right)\right)=0
$$


Evidently

$$
x_{n}\left(R \backslash\left(-\frac{1}{p}, \frac{1}{p}\right)\right)=x_{n}\left(\left(-\infty,-\frac{1}{p}\right]\right) \oplus x_{n}\left(\left[\frac{1}{p}, \infty\right)\right),
$$

because $R \backslash\left(-\frac{1}{p}, \frac{1}{p}\right)=\left(-\infty,-\frac{1}{p}\right] \cup\left[\frac{1}{p}, \infty\right)$ and therefore

$$
\bigvee_{n=k}^{\infty} x_{n}\left(R \backslash\left(-\frac{1}{p}, \frac{1}{p}\right)\right) \leq \bigvee_{n=k}^{\infty} x_{n}\left(\left(-\infty,-\frac{1}{p}\right]\right) \oplus \bigvee_{n=k}^{\infty} x_{n}\left(\left[\frac{1}{p}, \infty\right)\right)
$$

Hence

$$
\mathbf{m}\left(\bigvee_{n=k}^{\infty} x_{n}\left(R \backslash\left(-\frac{1}{p}, \frac{1}{p}\right)\right)\right) \leq \mathbf{m}\left(\bigvee_{n=k}^{\infty} x_{n}\left(\left(-\infty,-\frac{1}{p}\right]\right)\right)+\mathbf{m}\left(\bigvee_{n=k}^{\infty} x_{n}\left(\left[\frac{1}{p}, \infty\right)\right)\right)
$$

But

$$
\mathbf{m}\left(\bigvee_{p=1}^{\infty} \bigwedge_{k=1}^{\infty} \bigvee_{n=k}^{\infty} x_{n}\left(\left(-\infty,-\frac{1}{p}\right)\right)\right)=\mathbf{m}(\underline{x}((-\infty, 0)))=\mathbf{m}\left(0_{\mathcal{F}}((-\infty, 0))\right)=0 .
$$

On the other hand

$\mathbf{m}\left(\bigvee_{q=1}^{\infty} \bigvee_{k=1}^{\infty} \bigwedge_{n=k}^{\infty} x_{n}\left(\left(-\infty, \frac{1}{p}-\frac{1}{q}\right)\right)\right)=\mathbf{m}\left(\bar{x}\left(\left(-\infty, \frac{1}{p}\right)\right)\right)=\mathbf{m}\left(0_{\mathcal{F}}\left(\left(-\infty, \frac{1}{p}\right)\right)\right)=1$.

Since the last equality is equivalent with

$$
\lim _{q \rightarrow \infty} \mathbf{m}\left(\bigvee_{k=1}^{\infty} \bigwedge_{n=k}^{\infty} x_{n}\left(\left(-\infty, \frac{1}{p}-\frac{1}{q}\right)\right)\right)=1
$$

to each $\varepsilon>0$ there exists $q$ such that

$$
1-\varepsilon<\mathbf{m}\left(\bigvee_{k=1}^{\infty} \bigwedge_{n=k}^{\infty} x_{n}\left(\left(-\infty, \frac{1}{p}-\frac{1}{q}\right)\right)\right) \leq \mathbf{m}\left(\bigvee_{k=1}^{\infty} \bigwedge_{n=k}^{\infty} x_{n}\left(\left(-\infty, \frac{1}{p}\right)\right)\right) \leq 1
$$

Since the relation holds for every $\varepsilon>0$, then we have

$$
\mathbf{m}\left(\bigvee_{k=1}^{\infty} \bigwedge_{n=k}^{\infty} x_{n}\left(\left(-\infty, \frac{1}{p}\right)\right)\right)=1
$$

Hence by this equality holding for every $p \in N$ we obtain

$$
\mathbf{m}\left(\bigwedge_{p=1}^{\infty} \bigvee_{k=1}^{\infty} \bigwedge_{n=k}^{\infty} x_{n}\left(\left(-\infty, \frac{1}{p}\right)\right)\right)=1
$$

Finally using (10) we have

$$
\mathbf{m}\left(\bigvee_{p=1}^{\infty} \bigwedge_{k=1}^{\infty} \bigvee_{n=k}^{\infty} x_{n}\left(\left[\frac{1}{p}, \infty\right)\right)\right)=1-\mathbf{m}\left(\bigwedge_{p=1}^{\infty} \bigvee_{k=1}^{\infty} \bigwedge_{n=k}^{\infty} x_{n}\left(\left(-\infty, \frac{1}{p}\right)\right)\right)=1-1=0
$$

By (8), (9) and (11) we obtain (7), i.e., a sequence $\left(x_{n}\right)_{n}$ of an $I F$-observables converges m-almost everywhere to 0 .

In accordance to Proposition 4.1, we can extend the notion of $\mathbf{m}$-almost everywhere convergence by the following way. 
Definition 4.5. A sequence $\left(x_{n}\right)_{n}$ of IF-observables converges $\mathbf{m}$-almost everywhere to an IFobservable $x$, if

$$
\mathbf{m}\left(\bigvee_{p=1}^{\infty} \bigvee_{k=1}^{\infty} \bigwedge_{n=k}^{\infty} x_{n}\left(\left(-\infty, t-\frac{1}{p}\right)\right)\right)=\mathbf{m}\left(\bigvee_{p=1}^{\infty} \bigwedge_{k=1}^{\infty} \bigvee_{n=k}^{\infty} x_{n}\left(\left(-\infty, t-\frac{1}{p}\right)\right)\right)=\mathbf{m}(x((-\infty, t)))
$$

for every $t \in R$.

\section{Conclusion}

The paper is concerned in a modification of $\mathbf{m}$-almost everywhere convergence with the help of lim sup and lim inf and the construction of translations formulas for these limits in an intuitionistic fuzzy case. We compare two kinds of definition of the $\mathbf{m}$-almost everywhere convergence for intuitionistic fuzzy events and we show that they are equivalent.

\section{References}

[1] Atanassov, K. (1999). Intuitionistic Fuzzy Sets: Theory and Applications. Physica Verlag, Berlin.

[2] Atanassov, K. T. (2012). On Intuitionistic Fuzzy Sets. Springer, Berlin.

[3] Grzegorzewski, P., \& Mrówka, E. (2002). Probability of intuistionistic fuzzy events. In $P$. Grzegorzewski et al. eds, Soft Metods in Probability, Statistics and Data Analysis, Physica Verlag, New York, 105-115.

[4] Lendelová, K. (2007). Almost eweryvhere convergence in family of IF-events with product. In New Dimensions in Fuzzy Logic and Related Technologies: Procedings of the 5th EUSFLAT Conference, Ostrava, Czech Republic, 11-14 September 2007, 231-236.

[5] Riečan, B. (2003). A descriptive definition of the probability on intuitionistic fuzzy sets. In M. Wagenecht, R. Hampet eds., EUSFLAT'2003, Zittau-Goerlitz Univ. Appl. Sci., 263-266.

[6] Riečan, B. (2006). On a problem of Radko Mesiar: general form of IF-probabilities. Fuzzy Sets and Systems, 152, 1485-1490.

[7] Riečan, B. (2007). Probability theory on intuitionistic fuzzy events. In A volume in honour of Daniele Mundici's 60th birthday Lecture Notes in Computer Science.

[8] Riečan, B. (2012). Analysis of fuzzy logic models, Intelligent Systems (V. Koleshko ed.), INTECH, 219-244.

[9] Riečan, B. \& Neubrunn, T. (1997). Integral, Measure and Ordering. Kluwer, Dordrecht. 